\title{
Enquête
}

Archives de la revue Enquête

4 | 1988

Varia

\section{Un exemple de lisibilité dramaturgique}

L'organisation para-musicale des opéras italiens créés et diffusés à Milan entre 1840 et 1870

\section{Emmanuel Pedler}

\section{(2) OpenEdition}

Journals

\section{Édition électronique}

URL : http://journals.openedition.org/enquete/61

DOI : 10.4000/enquete.61

ISSN : 1953-809X

Éditeur :

Cercom, Éditions Parenthèses

Édition imprimée

Date de publication : 2 juin 1988

\section{Référence électronique}

Emmanuel Pedler, « Un exemple de lisibilité dramaturgique », Enquête [En ligne], 4 | 1988, mis en ligne le 27 juin 2013, consulté le 19 avril 2019. URL : http://journals.openedition.org/enquete/61 ; DOI :

10.4000/enquete.61

Ce document a été généré automatiquement le 19 avril 2019. 


\section{Un exemple de lisibilité dramaturgique}

L'organisation para-musicale des opéras italiens créés et diffusés à Milan entre 1840 et 1870

\section{Emmanuel Pedler}

1 Curieusement, le questionnement en sociologie de la réception, en partie étranger aux préoccupations musicologiques traditionnelles, conduit parfois sur les terres les plus arides de l'histoire musicale européenne, ces lieux à peine nommés où la pauvreté se masque. Le sociologue serait-il, plus que le musicologue, sensible aux mirages culturels ? Par esprit de contradiction? De fait, les rares moments d'intense création où les formes s'inventent et se renouvellent se prêtent mal à l'observation sociologique : qu'il soit mécène ou « lecteur » averti, le public, alors raréfié, se fond dans le milieu musical et ne dépose plus dans les greniers de l'histoire que la surface lisse, hors de prise, des commentaires. Et le sociologue de la musique, plus désorienté que ses confrères, doit se contenter de rares indices aux significations aléatoires. En effet, il faut se rendre à l'évidence, le flux musical, contrairement au texte et à l'image, interdit le commentaire détaillé ; seule la description musicologique savante a quelque prise, non sans mal; l'ethnomusicologue en a fait les frais et la transcription des formes élaborées, qu'elles soient polyrythmiques ou polyphoniques, opération difficile qui ne peut contourner le sens musical, doit se nourrir de la réception et mobiliser de subtiles techniques interactives $^{1}$. En conséquence la sociomusicologie ${ }^{2}$ est-elle contrainte à la contemporanéité ? Doit-elle penser son objet sans repères historiques ? Elle le doit, à moins qu'elle ne porte, précisément, son attention sur les terres inconnues de la musicologie traditionnelle, ces époques où le public peu formé et indifférent élit des formes hybrides dans lesquelles s'impriment des cheminements fléchés, des découpages possibles et pensables auxquels le commentaire peut s'accrocher. Et du même coup, l'étude des pratiques musicales contemporaines se voit dotée d'instruments qu'elle n'aurait pu improviser ex abrupto. Les riches heures de l'histoire musicale savante, les moments où s'inventent la polyphonie contrapuntique ou l'harmonie, le madrigal, la 
fugue ou la symphonie n'ont pas laissé les traces prosaïques des périodes obscures où fleurit l'opéra.

2 L'opéra romantique italien ne favorisait pas, historiquement et sémiotiquement, une trajectoire allant de la littérature vers la musique, ni même l'inverse. Il est plus exact de noter que par sa forme et par la matière qui l'alimentait, il était lui-même le plus court chemin qui mène à la littérature et à la musique. Mais comment ce genre semi-cultivé assurait-il son accessibilité? Si ces opéras étaient, en quelque sorte, la vitrine de la création littéraire et musicale européenne, une sorte d'« Apostrophes » en acte, comment facilitaient-ils leur propre lisibilité face à un public peu formé musicalement et qui, du reste, ne pouvait accéder qu'à de très rares traductions? La réponse traditionnelle ne saurait nous convaincre : la prétendue stéréotypie dramaturgique des livrets se conjugue mal avec la grande diversité et l'abondance de leurs sources littéraires. La surface hétérogène des œuvres (ce sont des messages multiples) où des marques para-musicales flèchent quelques parcours possibles, doit être auscultée plus finement.

\section{Une sociologie historique des musiques impures}

\section{a) Problématique générale}

3 Le mouvement qui anime les musiques savantes conduit sporadiquement au «décrochage» de ces musiques de tout ancrage extra-musical: texte et narration, organisation quasi narrative des développements, expériences hyperesthésiques extramusicales, ancrage gestuel, etc. Si l'on en croit la musicologie traditionnelle, la somme de ces œuvres musicales "pures ", dès lors qu'elle est " contextualisée » par la description historique des moments d'élaboration et de création des œuvres, devrait constituer l'histoire musicale européenne. Curieuse illusion! La partialité d'une telle démarche est plus manifeste encore lorsqu'il est question d'histoire des publics et des pratiques musicales, comme en témoigne l'un des seuls textes de synthèse écrit à ce sujet, Histoire du public musical de R. Siohan ${ }^{3}$, conçu à partir de la même doctrine transportée sur un autre terrain. L'auteur propose une « coupe » homogène sur une très longue durée dont les séquences trahissent l'incohérence du projet: qu'il y ait des moments d'éclipse de la création musicale savante peut se concevoir, que des populations de spectateurs puissent disparaître sans laisser de traces est plus curieux. Néanmoins ces raccourcis, en dépit de leur faiblesse, laissent émerger un constat encore fragile qui n'est pas sans intérêt : les seuls moments de diffusion de cette musique "pure", lorsqu'elle ne prend pas une dimension spéculative et marginale ou qu'elle n'est pas artificiellement entretenue ${ }^{4}$, se situent en ces rares périodes où la pratique instrumentale du public - au sens large du terme-, donc son niveau de formation musicale, a pu connaître le plus fort développement.

4 À la lumière de ces remarques, on peut s'attendre à observer, dans l'histoire musicale européenne, des dénivellations sensibles dans la formation musicale des publics lors des périodes d'éclipses des formes savantes. Ce fut le cas, semble-t-il, au moment où le public aristocratique, souvent plus cultivé que le public bourgeois, dut quitter la scène publique. Une illustration de cette configuration historique probable se rencontre en France, durant la seconde moitié du XVIII ${ }^{\mathrm{e}}$ siècle. L'édition musicale témoigne, en effet, durant la première partie du demi-siècle, d'un important accroissement de la pratique instrumentale aristocratique ${ }^{5}$ à laquelle répond l'édification de la « symphonie française » 
(Le Duc, Gossec, Martin, chevalier de Saint-Georges, etc.). Au tournant du XIX ${ }^{e}$ siècle, la symphonie laisse la place à l'opéra, consommé par un public peu formé. L'édition de partitions de symphonies ${ }^{6}$ ou de symphonies concertantes s'éteint au profit de "potspourris » tirés d'opéras, dont les « résumés » pianistiques peuvent se lire sans trop de difficulté?.

Quelques décennies plus tard, l'édition musicale présente en Italie les mêmes caractéristiques. La formation musicale élémentaire - prodiguée essentiellement aux femmes - ne permet que la lecture, probablement approximative, de "pots-pourris » tirés d'opéras.

6 Le cours le plus fréquent de l'histoire du public musical semble présenter à chaque fois un même profil : au moment où la formation musicale se dégrade, une prédilection pour les formes moins élaborées se fait jour ; or ces formes tendanciellement recourent soit à la chanson sous toutes ses formes (dans la première moitié du XIX siècle en France, la romance, dont la diffusion massive est attestée par l'édition, s'impose durablement), soit à l'opéra qui, sauf exception, est toujours resté une forme musicale mineure.

7 Prolongeant cette interrogation, il devenait nécessaire de poser, dans les mêmes termes, la question des modes d'accès à la musique utilisés de nos jours, pour un public encore moins formé qu'au XIX ${ }^{e}$ siècle. Or, les conclusions convergent: les premiers résultats d'une enquête menée actuellement ${ }^{8}$ sur les choix multiples (8 à 10 œuvres) effectués par une population très cultivée mais inégalement formée musicalement $(\mathrm{N}=120)$ nous indiquent que moins les sujets sont musicalement cultivés (absence d'apprentissage instrumental), plus ils choisissent d'œuvres vocales (opéras, mélodies, oratorios...). En outre, on observe la même liaison entre forme courte (mélodies, pièces instrumentales courtes, mouvements les plus courts d'une œuvre longue) et absence de formation musicale. Enfin, si le commentaire détaillant une pièce longue instrumentale (pour l'apprécier, l'interpréter ou la décrire en sa forme) est très rare et ne se rencontre que chez les individus musicalement cultivés, la même opération est fréquente, à partir du texte, sur des œuvres lyriques, chez les sujets les moins formés musicalement. L'accès aux œuvres lyriques - musique « impure » par excellence -, est la moins savante des pratiques musicales savante ; il s'agit d'une maîtrise lettrée de l'écoute musicale?.

$8 \mathrm{Au}$ reste, on peut s'attendre à la disparition de cette modalité littéraire de l'accès aux œuvres chez les sujets peu diplômés et musicalement peu cultivés, sans que, pour autant, la prédilection pour les œuvres vocales et/ou courtes disparaisse. De fait, dans l'accès « littéraire » aux œuvres coexistent deux dimensions distinctes: d'un côté l'activité de repérage, d'indexation ${ }^{10}$, de l'autre le supplément d'«âme " littéraire et esthétique que tend à insuffler le dilettante lettré à l'œuvre musicale.

Ces premières conclusions nous ont naturellement conduit à placer les œuvres musicales organisées comme des "messages multiples", selon l'expression de A. Moles, au centre de nos recherches. Tenter une sociologie historique des musiques «impures» est certainement aux yeux de la musicologie une tâche dérisoire, mais c'est la seule perspective qui soit empiriquement crédible.

10 Pourtant, bien que la musique "impure ", toujours sensible à l'enjeu de sa propre lisibilité, soit la plus accessible à l'analyse socio-musicologique, il est, de manière générale, très difficile de proposer des descriptions de la réception modale de ces genres hybrides qui puissent se contrôler empiriquement. Rien n'assure que la partie littéraire de l'opéra constitue toujours une armature pour la réception musicale des œuvres 
lyriques, il est même fortement probable que dans bien des cas des livrets issus de textes littéraires n'aient jamais eu d'autre fonction que de servir de guide aux compositeurs - c'est surtout le cas à des époques où l'on n'écoute jamais l'œuvre musicale dans son intégralité ${ }^{11}$. Comment s'assurer, sinon indirectement, de l'importance «littéraire » de l'opéra? Ainsi, si tous les moments d'éclipse des formes les plus savantes sont également des terrains possibles pour tester notre hypothèse, seules certaines configurations sont réellement susceptibles, par la clarté de leur profil, de faire avancer la discussion.

Le choix du « cas italien » tient à plusieurs raisons :

- La formation musicale instrumentale du public est faible, c'était une condition nécessaire.

- L'hégémonie, sans concurrence, de l'opéra durant près de soixante-dix ans sur les scènes italiennes permet de mieux contrôler les caractéristiques des publics ${ }^{12}$ et les modalités de leurs comportements : nos sources sont naturellement plus abondantes ici qu'ailleurs.

- La très grande diversité des sources littéraires des opéras signale un important travail des librettistes sur un «canal » qui a pu, à d'autres époques, véhiculer des poncifs ou des textes littérairement insignifiants pour le public. La marginalité économique et culturelle de l'Italie par rapport au reste de l'Europe, ainsi que les caractéristiques du public modal des théâtres lyriques confirment la place centrale occupée par le livret d'opéra dont la fonction de vitrine de la culture littéraire européenne est originale.

- L'activité considérable des théâtres lyriques tant au niveau de la création (il s'agit d'un secteur économique rentable qui favorise largement la création jusqu'en 1860), que de la diffusion (il n'existe pas de répertoires stabilisés mais les œuvres circulent) fait de cette époque un terrain privilégié de l'observation socio-historique.

\section{b) L'exemple italien et la vie musicale milanaise de 1840 à 1870}

12 Avant d'en venir à l'exemple milanais qui nous permettra d'entrer dans un détail qu'interdisent naturellement les vastes panoramas, il n'est pas inutile de fixer à grands traits les caractéristiques générales de la vie musicale italienne.

Est-il une meilleure introduction que le récit que nous fit de ses pérégrinations H. Berlioz, voyageur attentif du début du siècle? Son expertise musicale ne saurait être contestée, elle est précise, détaillée et reste vive et d'un ton qui engage à la lecture, ce qui ne gâte rien. Ce musicien, hôte de la Villa Médicis en 1830 et 1831, consacra une centaine de pages ${ }^{13}$ ses voyages dans les États italiens ${ }^{14}$. Florence, Rome, Naples, Milan sont ses principales étapes. Remarquons que l'abondance de la création et de la diffusion d'opéras en Italie reçoit ici une illustration concrète : voyageur pédestre, du reste peu argenté, $\mathrm{H}$. Berlioz peut écouter sept opéras nouveaux lors de ses excursions hors de la Villa Médicis. Il commente vivement. Suivons-le :

« De toutes les capitales d'Italie, aucune ne m'a laissé d'aussi gracieux souvenir que Florence [...] Sachant bien que je ne trouverais pas dans la capitale de la Toscane ce que Naples ou Milan me faisaient tout au plus espérer, je ne songeais guère à la musique, quand les conversations de table d'hôte m'apprirent que le nouvel opéra de Bellini (I Montecchi e i Capuletti) allait être représenté. On disait beaucoup de bien de la musique, mais aussi beaucoup du libretto, ce qui, eu égard au peu de cas que les Italiens font pour l'ordinaire des paroles d'un opéra, me surprenait étrangement [... ] Je courus au théâtre de la Pergola. Les choristes nombreux qui couvraient la scène me parurent assez bons; leurs voix sonores et mordantes; il y avait surtout une douzaine de petits garçons de quatorze à quinze ans, dont les contralti étaient d'un excellent effet. Les personnages se présentèrent successivement et chantèrent tous faux, à l'exception de deux femmes, dont l'une, grande et forte, remplissait le rôle de 
Juliette, et l'autre, petite et grêle, celui de Roméo. Pour la troisième ou quatrième fois après Zingarelli et Vaccai, écrire encore Roméo pour une femme... [...] Décidé à boire le calice jusqu'à la lie, je voulus, quelques jours après, entendre La Vestale de Paccini. Quoique ce que j'en connaissais déjà m'eût bien prouvé qu'elle n'avait de commun avec l'œuvre de Spontini que le titre, je ne m'attendais à rien de pareil... Licinius était encore joué par une femme... Après quelques instants d'une pénible attention, j'ai dû m'écrier, comme Hamlet: "Ceci est de l'absinthe!" et ne me sentant pas capable d'en avaler davantage, je suis parti au milieu du second acte [...] Pauvre Italie !... on verra plus tard quelle musique on fait à Rome, dans la capitale du monde chrétien ${ }^{15}$.»

Et quelques pages plus loin :

«Les chanteurs que j'ai entendus pendant la saison théâtrale [à Rome] avaient en général de bonnes voix et cette facilité de vocalisation qui caractérise spécialement les Italiens, mais, à l'exception de Mme Ungher, prima donna allemande, que nous avons applaudie souvent à Paris et de Salavator, assez bon baryton, ils ne sortaient pas de la ligne des médiocrités, les chœurs sont d'un degré au-dessous de ceux de notre Opéra-Comique pour l'ensemble, la justesse et la chaleur.

L'orchestre, imposant et formidable, à peu près comme l'armée du prince de Monaco, possède, sans exception, toutes les qualités qu'on appelle ordinairement des défauts. Au théâtre Valle, les violoncelles sont au nombre de... un, lequel exerce l'état d'orfèvre, plus heureux que l'un de ses confrères, obligé, pour vivre, de rempailler des chaises ${ }^{16}$. À Rome, le mot symphonie, comme celui d'ouverture, n'est employé que pour désigner un certain bruit que font les orchestres de théâtre, avant le lever de la toile, et auquel personne ne fait attention. Weber et Beethoven sont là des noms à peu près inconnus. Un savant abbé de la chapelle Sixtine disait un jour à Mendelssohn qu'on lui avait parlé d'un jeune homme d'une grande espérance nommé Mozart [...] Il faut, on le voit, renoncer à peu près à entendre de la musique, quand on habite Rome ${ }^{17}$. "

H. Berlioz se montre moins sévère lorsqu'il décrit son passage au San Carlo de Naples : « ... là pour la première fois depuis mon arrivée en Italie, j'entendis de la musique. L'orchestre, comparé à ceux que j'avais observés jusqu'alors, me parut excellent. Les instruments à vent peuvent être écoutés en sécurité ; on n'a rien à craindre de leur part; les violons sont assez habiles, et les violoncelles chantent bien, mais ils sont en trop petit nombre [...] Je reprocherais bien aussi au maestro di capella le bruit souverainement désagréable de son archet dont il frappe un peu rudement son pupitre ; mais on m'a assuré que sans cela, les musiciens qu'il dirige seraient quelque fois embarrassés pour suivre la mesure... À cela il n'y a rien à répondre ; car enfin, dans un pays où la musique instrumentale est à peu près inconnue, on ne doit pas exiger des orchestres comme ceux de Berlin, de Dresde ou de Paris. Les choristes sont d'une faiblesse extrême; je tiens d'un compositeur qui a écrit pour le théâtre Saint-Charles, qu'il est fort difficile, pour ne pas dire impossible, d'obtenir une bonne exécution des chœurs écrits à quatre parties. Les soprani ont beaucoup de peine à marcher isolés des ténors, et on est pour ainsi dire obligé de les leur faire continuellement doubler à l'octave ${ }^{18}$. "

Le voyage musical de $\mathrm{H}$. Berlioz se termine à Milan :

«En arrivant à Milan, il fallut, pour l'acquit de ma conscience, aller voir le Nouvel Opéra. On jouait alors à la Cannobiana l'Elisir d'amore de Donizetti. Je trouvai la salle pleine de gens qui parlaient tout haut et tournaient le dos au théâtre ; les chanteurs gesticulaient toutefois et s'époumonaient à qui mieux mieux [...] On jouait, on soupait dans les loges, etc., etc. [...] En tout cas, la musique pour les Milanais, comme pour les Napolitains, les Romains, les Florentins ou les Génois, c'est un air, un duo, un trio, tels quels, bien chantés ; hors de là ils n'ont plus que de l'aversion ou de l'indifférence [...] De tous les peuples de l'Europe, je penche fort à le regarder comme le plus inaccessible à la partie poétique de l'art ainsi qu'à toute conception excentrique un peu élevée. La musique n'est pour les Italiens qu'un plaisir des sens, rien d'autre [...] Ils veulent des partitions dont ils puissent du premier coup, sans 
réflexion, sans attention même, s'assimiler la substance ; comme ils feraient d'un plat de macaroni ${ }^{19}$.»

Il ne fait pas de doute qu'entre 1830, date à laquelle écrit Berlioz, et 1840, moment où nous nous situons, de sensibles modifications aient affecté la vie musicale italienne. Déjà, à la fin du XVIII ${ }^{\mathrm{e}}$ siècle, un certain nombre de compositeurs italiens s'exilent, en France notamment; le tissu musical se distend et perd peu à peu en consistance. Assiste-t-on, à l'image de ce que décrit B. S. Brook pour la France, à une transformation équivalente des publics $^{22}$ ? W. Weber ${ }^{23}$ parle pour la France, l'Allemagne et l'Angleterre de l'apparition d'un public composé d'une middle class peu cultivée musicalement. En est-il de même en Italie? Cela est fort probable.

Sur les scènes des grandes villes italiennes, la musique vocale connaîtra en quelques années un essor considérable. De 1825 à 1850, plus d'un millier d'opéras sont créés ${ }^{24}$. La réorganisation et la gestion des théâtres lyriques deviennent alors une opération profitable qu'organisent des entrepreneurs actifs. Parallèlement, l'Italie reste, en matière d'enseignement musical, une véritable terre brûlée de la formation savante et le volontarisme constructiviste des entrepreneurs ne peut expliquer à lui seul l'émergence d'une culture vocale dont la complexité ira croissant et qui occupera sans partage toute la scène musicale. Les cultures vocales populaires italiennes ont-elles exercé indirectement une influence sur l'élaboration de ce que l'on a appelé l'« opéra romantique italien » ? En tout cas, les acteurs principaux de cette édification, les compositeurs et les chanteurs, seront dans l'ensemble des autodidactes dont la culture vocale doit probablement beaucoup plus au milieu d'origine qu'aux rudiments mal intériorisés de la musique savante ${ }^{25}$. Toujours est-il qu'en quelques années émerge un nouveau type d'opéra, caractérisé par l'abondance et la consistance des œuvres créées, le centrage autour de 
villes jusque-là beaucoup moins actives (les anciens bastions comme Naples ou Venise perdent en influence) et enfin et surtout par une forme musicale et théâtrale renouvelée.

Le flux des œuvres créées et diffusées à Milan de 1778 à 1872 semble, au premier regard régulier ${ }^{26}$. Il y eut de 10 à 20 opéras donnés à la Scala chaque année, avec un net infléchissement dans les années 1860 . Pourtant cette série n'est pas homogène. On assiste dans les années 1840 à la disparition progressive des opéras buffa, semi-seria et desfarza (tableau 1).

Tableau 1. Nombre d'opéras diffusés selon les « genres »

\begin{tabular}{|l|c|c|c|c|c|c|c|c|}
\hline Perriode & $1820-24$ & $1825-29$ & $1830-34$ & $1835-39$ & $1840-44$ & $1845-49$ & $1850-54$ & $1855-59$ \\
\hline Serio" & 25 & 42 & 32 & 41 & 67 & 48 & 37 & 53 \\
Buffo" $^{\circ}$ & 43 & 37 & 21 & 28 & 26 & 12 & 8 & 5 \\
\hline Total & 68 & 79 & 53 & 69 & 93 & 60 & 45 & 58 \\
\hline
\end{tabular}

"Serio et sacro. "* Buffo, semi-serio et farza.

20 Or la construction d'un opéra seria et celle d'un opéra buffa diffère habituellement, au moins par la longueur. Surtout, les opéras séria, dans les années 1840 changent de facture, ils tendent à s'allonger et requièrent de plus en plus de travail de la part du compositeur et du librettiste ${ }^{27}$.

21 Malgré la continuité apparente de la production d'opéra dans cette première moitié du $\mathrm{XIX}^{\mathrm{e}}$ siècle, et indépendamment de toute classification a priori en genres distincts, un renouvellement très sensible se fait jour ${ }^{28}$.

Il est difficile de qualifier ce nouveau type d'opéra. Aucun terme spécifique n'a été jusqu'ici affecté à cet ensemble qui est historiquement et socialement très homogène bien qu'il se range formellement dans un "genre" qui recoupe, de fait, des objets très dissemblables quant à leur utilisation modale. Nous parlerons d'« opéra romantique italien » sans reprendre à notre compte ce que sous-tend cette qualification dans les classifications musicologiques.

\section{d) Une réception modale non lettrée}

23 Le niveau de formation musicale de nos contemporains a, d'une manière générale, sensiblement baissé au regard de la formation, pourtant élémentaire, de la middle class qui fréquentait les théâtres lyriques au XIX ${ }^{\mathrm{e}}$ siècle. La modalité très lettrée - au sens propre du terme - de l'accès des groupes les plus diplômés aux œuvres musicales, qu'elles soient lyriques ou même instrumentales, est une caractéristique moderne. Or on peut dire que l'«opéra romantique italien " se situe à l'opposé de cette figure, c'est même là un caractère qui le distingue de ses homologues contemporains. En un mot on ne peut pas plus le décrire comme genre littéraire ou théâtral autonome que comme genre musical original.

En effet, les livrets ont, pour partie, vulgarisé la littérature européenne (allemande, espagnole, française, anglaise). F. Dutheil a souligné29 la faiblesse de la traduction littéraire en Italie vers le milieu du siècle ; pourtant Shakespeare, Schiller, Byron, Hugo, Dumas, Scott et d'autres seront connus du public par les livrets régulièrement publiés et largement diffusés ${ }^{30}$. À titre indicatif, l'année 1859 donne une image moyenne des représentations habituellement offertes aux spectateurs. 
Tableau 2. Origine des livrets des opéras seria donnés en 1859 à la Scala

\begin{tabular}{|llcl|}
\hline \multicolumn{1}{|c}{ Titre } & Compositeur & $\begin{array}{c}\text { Date de } \\
\text { création }\end{array}$ & Auteur adapté \\
\hline Vasconcello & Villanis & 1852 & \\
Semiramide & Rossini & 1823 & Voltaire \\
Simon Boccanegra & Verdi & 1857 & Gutièrrez (Esp.) \\
Norma & Bellini & 1831 & L. A. Souma (F) \\
Maria de' Ricci & F. Asioli & création & \\
Il crociato in Egitto & Meyerbeer & 1824 & \\
II duca di Scilla & Petrella & création & \\
Il trovatore & Verdi & 1853 & Gutièrrez \\
Lucia di Lammermoor & Donizetti & 1835 & W. Scott \\
Il giuramento & Mercadante & 1837 & \\
Rigoletto & Verdi & 1851 & Hugo \\
Matilde de Shabran & Rossini & 1851 & \\
Riccardo III & Meiners & 1859 & Shakespeare \\
Gli Ugonotti & Meyerbeer & 1836 & Deschamps \\
\hline
\end{tabular}
narration avec un intérêt autrement aiguisé que le nôtre: on peut supposer en conséquence que l'attention qu'il portait à la partie littéraire de l'œuvre devait être plus aisément captée, malgré l'inintelligibilité traditionnelle du texte chanté. Les conditions 
matérielles d'exécution, le manque d'homogénéité du texte chanté selon les registres, la densité très variable des livrets ${ }^{33}$, la versification des textes et les archaïsmes littéraires nombreux contribuaient certainement à interdire une lecture directe de l'œuvre; la déperdition d'informations devait être, pour l'écoute directe, considérable. Enfin l'hétérogénéité des sources (romans, pièces de théâtres, nouvelles, de toutes nationalités) ne garantissaient pas la transparence d'une organisation narrative toujours renouvelée.

Même si l'on suppose que le public, qui du reste ne faisait la plupart du temps qu'une seule «lecture » de l'œuvre, pouvait régulièrement anticiper le spectacle par la lecture des livrets, le problème demeure, pour l'essentiel. En effet la pré-connaissance des grandes lignes de la narration permet un «balisage » sommaire de l'œuvre mais ne donne pas accès à l'économie narrative, souvent complexe, activée par les interprètes. En un mot, indépendamment de la pré-connaissance du tissu narratif, se pose la question du suivi in situ.

Deux hypothèses sont envisageables : a) le filtre du livret, la simplification littéraire et l'amplification des oppositions qu'il favorise conduiraient à une stéréotypie dramaturgique ; b) l'accessibilité de l'œuvre tiendrait à d'autres caractéristiques infralittéraires et infra-musicales assurant une " signalisation narrative ».

Le commentaire essayiste qui fleurit autour de l'opéra italien affirme aujourd'hui que ce dernier est organisé autour d'une dramaturgie stéréotypée qui en assure l'accessibilité. Il s'agit d'une erreur de perspective amplifiée par l'exiguïté extrême du nombre des œuvres qui servent à étayer ce jugement (les classements qui ont cours n'ont pas retenu plus de $2 \%$ des œuvres d'origine). De fait, les stéréotypes ne sont pas absents, mais leur combinatoire est relativement complexe ${ }^{34}$. Autrement dit, les éléments constitutifs de la dramaturgie sont connus séparément, mais les constructions qui les chaînent sont à chaque fois inédites, ce qui ne fournit aucune aide au suivi de l'œuvre. D'ailleurs, il ne pouvait en être autrement, les règles qui conduisent à filtrer le texte d'origine pour construire un livret sont terriblement contraignantes : il s'agit de réduire une narration multipolaire (organisée autour d'un grand nombre de personnages) à trois protagonistes aux profils très dessinés. Ainsi, si l'on prend toujours des personnages comparables, aucun stéréotype fonctionnel (au sens où le conflit mélodramatique entre mari trompé, femme adultère et amant est un construit stéréotypé fonctionnel) ne s'impose incontestablement et durablement.

33 Mais il existe pourtant des régularités. Les personnages exercent, dans la narration, des fonctions souvent très différentes, mais la structure du récit leur attribue des places fixes ou positions conventionnelles. Nous entendons par positions conventionnelles des places ou pôles au nombre stable appelées à être occupées par des personnages dont les fonctions peuvent varier et qui, conventionnellement, sont toujours identifiées. Dans l'«opéra romantique italien", les trois personnages principaux sont conventionnellement désignés : deux, de même sexe, se distinguent toujours entre eux par des marques para-musicales, ils s'opposent au troisième par le sexe. Mais n'anticipons pas: existe-t-il pour tout le monde des personnages principaux? Sont-ils toujours identifiés? Par quel moyen?

\section{b) La pré-désignation conventionnelle}

34 La notion de héros telle que P. Hamon l'a modélisée est sur cette question un bon point de départ. Délaisser les instruments sophistiqués de la sémiotique (les actants par exemple) 
pour élire le concept commun de « personnage » ne répond pas à une démarche naïve ou provocatrice ${ }^{35}$.

Dans l'opéra, en raison du resserrement de la narration autour de quelques personnages, l'utilisation d'un tel concept s'impose encore plus qu'ailleurs. La question la plus difficile est de déterminer les personnages centraux.

P. Hamon définit un certain nombre de constantes :

- Une qualification préalable différentielle.

- Une distribution différentielle (apparition des personnages).

- Une autonomie différentielle (par exemple, lorsque tous les personnages apparaissent accompagnés et le héros toujours seul).

- Une fonctionnalité différentielle (allusion aux travaux de Propp).

- Une pré-désignation conventionnelle (liée au genre).

En un mot, différents filtres simplifient le travail de reconnaissance du spectateur.

Remarquons l'hétérogénéité du statut sémiotique de ces constantes. Elles se matérialisent par des marques linguistiques ou extra-linguistiques. Il s'agit d'une distinction que P. Hamon ne pose pas d'emblée pour un raison simple: le matériel sur lequel il raisonne couramment est littéraire, donc toujours linguistique. Pour nous la distinction est importante. Qu'il s'agisse de l'autonomie différentielle des personnages ou de la prédésignation conventionnelle des positions occupées par ces mêmes personnages, les marques non linguistiques sont, dans l'opéra, très fréquentes. Il faudrait même ajouter une autre distinction opposant marques linguistiques internes (du livret) et marques linguistiques externes (qui émanent de supports divers contribuant à familiariser le spectateur potentiel, annonces, invitations circonstanciées, etc.).

Notre première question va au-delà de la pré-catégorisation proposée par P. Hamon, tout en bénéficiant des distinctions qu'il pose. La question est bien celle-ci : quels sont les filtres successifs qui simplifient graduellement le travail du spectateur?

La pré-désignation conventionnelle joue ici un grand rôle. P. Hamon définit cette prédésignation conventionnelle de la façon suivante :

«Le genre fonctionne comme un code, commun à l'émetteur et au récepteur, qui restreint et prédétermine l'attente de ce dernier en lui imposant des lignes de moindre résistance (prévisibilité totale). Ainsi, la commedia dell'arte, l'opéra, le western, etc., l'emploi de masques, de costumes, d'un type de phraséologie, de modalités d'entrée en scène, etc. fonctionnent comme autant de marques désignant d'emblée le héros, pour qui possède la grammaire du genre ${ }^{36}$."

Néanmoins, ce fléchage n'a de sens que pour les trois personnages principaux. Un premier tri est opéré par des voies très diverses allant de la connaissance des noms propres des interprètes principaux à la lecture des comptes rendus, annonces diverses. Dans tous les cas, l'autonomie différentielle des personnages, ici leur présence effective, vient renforcer ce premier tri. Par exemple, dans le Macbeth (version de 1865), une dizaine de personnages sont nominalement individualisés et présentés comme tels sur scène : Lady Macbeth, Macbeth, Banquo, Macduff, Malcolm, une suivante, un messager, un serviteur, un médecin. Néanmoins, l'autonomie différentielle des personnages principaux se marque par une présence dont la longueur contraste avec le « passage » des petits rôles: dans l'ordre 32', 34', 6', 2', 1' et trois temps inférieurs à la minute ${ }^{37}$. L'opposition nom propre $v s$ nom générique (Macbeth $v s$ un médecin, par exemple) corrobore exactement la sélection de l'affiche qui isole les "rôles secondaires". Néanmoins, les temps, les noms propres et les rôles annoncés ne permettent qu'un tri 
grossier. Il est néanmoins très remarquable que le filtre du livret effectue justement ce reserrement autour de trois personnages alors que le texte d'origine peut utiliser un nombre très variable de personnages principaux.

Le second tri qui peut avoir lieu entre trois à cinq personnages en isole trois reliés entre eux par une double opposition que l'on peut figurer ainsi :

\begin{tabular}{|lccc|}
\hline Personnage & P 1 & P 2 & P 3 \\
Sexe (également marqué vocalement) & $\mathrm{H}$ & $\mathrm{F}$ & $\mathrm{H}$ \\
& $\mathrm{F}$ & $\mathrm{H}$ & $\mathrm{F}$ \\
Tessiture & + grave & & + aigu \\
\hline
\end{tabular}

\section{c) Les registres vocaux, un marquage para-musical}

43 La pré-désignation conventionnelle passe, on l'a déjà dit, par des marques vocales et sexuelles. Ainsi, dans l'opéra, la partition vocale (opposition des classes tessiturales) joue le rôle des masques de la commedia dell'arte. En particulier les positions conventionnelles sont indépendantes des fonctions, nous l'avons déjà souligné. Le bon mot habituellement attribué à $\mathrm{B}$. Shaw selon lequel un opéra (italien $\mathrm{du} \mathrm{XIX}^{\mathrm{e}}$ siècle) se résume de la manière suivante: «Un ténor et une soprano veulent coucher ensemble et un baryton les en empêche " n'est exacte que pour moitié. Les fonctions peuvent varier (modalité des interactions entre les personnages) tout en s'organisant autour de ces positions conventionnelles. Il vaut donc mieux dire que trois personnages P 1, P 2, P 3 sont doublement opposés par le sexe et la tessiture : P 1 et $\mathrm{P} 3$ sont de même sexe mais chantent dans une tessiture différente, $\mathrm{P} 2$ est de sexe différent. Cette double opposition se signale complètement par les tessitures bien qu'elle soit également renforcée par d'autres marques; elle est donc non linguistique et de surcroît para-musicale puisqu'elle est extérieure à l'idiolecte musical.

L'axe sémantique des hauteurs de voix permet de caractériser précisément deux personnages-type. $\mathrm{P} 3$ possède une voix plus aiguë, $\mathrm{P} 1$ une voix plus grave. Mais cet axe n'est sémantique que pour autant que deux conditions sont réunies : il faut que dans la pratique (et la théorie éventuellement) des classes tessiturales soient opposées et il est nécessaire que le public connaisse et fasse signifier ces oppositions ${ }^{38}$.

L'organisation systématique des registres semble relativement récente. Les six classes tessiturales que l'on distingue depuis la seconde moitié du XIX siècle, à partir d'un découpage en tierces et octaves, forment un enchaînement allant du soprano à la basse. Ce sont les suivantes : soprano, mezzo-soprano, contralto, ténor, baryton, $1^{\text {re }}$ et $2^{e}$ basse (les voix en italique donnent les divisions principales) ${ }^{39}$. Dans l'histoire de l'opéra italien, et donc dans l'enseignement vocal qui lui est relié, l'amplitude maximale du nombre des voix distinctes réellement utilisées et travaillées fut atteinte dans la seconde moitié du XIX ${ }^{e}$ siècle. Néanmoins, le classement des registres opératoires, c'est-à-dire insérés dans des couples d'oppositions, ne constitue qu'un chapitre dans l'histoire des classements vocaux. Il faut certainement distinguer l'opéra des musiques vocales "pures ", c'est-à-dire des musiques qui ne sont pas réellement des «messages multiples» i.e. qui n'utilisent pas le texte à plein. L'originalité de l'« opéra romantique italien » tient à la modification de la pré-désignation conventionnelle tout entière traduite vocalement. Ce qui, dans l'opéra rossinien (pour utiliser des exemples connus), passait par la manifestation scénique et linguistique de "caractères ", est codé directement dans l'opposition de registres plus 
nombreux. Ainsi, dans l'Italiana in Algeri, la voix du Bey (basse) s'oppose bien à la voix de ténor de Lindoro, néanmoins en l'absence de systématicité, cette opposition ne suffit pas à les distinguer. Il existe de nombreux cas où deux basses « s'opposent », le Turc en Italie par exemple. La simple «lecture » des tessitures ne nous informe pas directement des grandes lignes de l'organisation de l'opéra. À noter que la subdivision fine des tessitures et leur utilisation organisée permettent l'économie de préambules détaillés, de récitatifs auparavant obligés et d'intermèdes.

La « traduction » vocale au travers de l'opposition des classes tessiturales n'est cependant en rien un code qui signifierait l'existence de positions stabilisées définitivement évaluées. Ainsi la formule de B. Shaw est doublement fausse. Pour faire appel aux seuls opéras très connus, ni Nabucco, ni Aïda ne correspondent au stéréotype vocal qu'il pointe. Ce sont les couples d'opposition qui déterminent les personnages-type. L'opposition baryton (Ba.)-ténor (T.) peut devenir l'opposition mezzo-soprano (M.S.) vs soprano (S.). Il suffit que $P 1$ et $P 3$ aient les mêmes sexes.

Un exemple d'équivalence

\section{Le bal masqué Renato (Ba.) Amelia (S.) \\ Riccardo (T.) Aida Amnéris (M.S.) Radamès (T.) Aïda (S.)}

47 La pré-désignation conventionnelle ne s'arrête pas au noyau narratif, c'est-à-dire aux trois personnages principaux. Entre les rôles principaux et les petits rôles, il existe une position intermédiaire. Si les personnages P 1, P 2, P 3 concentrent la plus grande part de la narration, les personnages intermédiaires sont utilisés afin de clarifier cette dernière. Ainsi en est-il des personnages anaphores qui

« tissent dans l'énoncé un réseau d'appels et de rappels à des segments d'énoncés disjoints et de longueur variable (un syntagme, un mot, un paragraphe...). Éléments à fonction essentiellement organisatrice et cohésive, ils sont en quelque sorte les signes mnémotechniques du lecteur: personnages de prédicateurs, personnages doués de mémoire, personnages qui sèment ou interprètent des indices, etc. ${ }^{40}$. "

Alors que les personnages secondaires peuvent être indifféremment soprano, ténor, baryton ou basse sans relations directes avec le noyau narratif, les personnages intermédiaires sont situés vocalement par rapport aux personnages principaux. Ainsi, les personnages anaphores, lorsqu'ils sont féminins, sont souvent très contralto ou mezzosoprano alors que le personnage $\mathrm{P} 2$ est une soprano.

Cette signalisation narrative prend néanmoins une forme quelque peu inhabituelle si on la compare à d'autres modèles narratifs. La focalisation et le grossissement des oppositions suggèrent l'existence d'une parenté avec l'organisation narrative du conte ${ }^{41}$. Pourtant, au-delà cette parenté, une différence essentielle oppose les deux formes: l'« opéra romantique italien" semble, spécifiquement, utiliser des positions conventionnelles indépendamment des actualisations fonctionnelles.

\section{Conclusion}

L'impossibilité de classer de façon non arbitraire les quatre, cinq personnages principaux interdit généralement un commentaire comparé rigoureux. La critique paramusicologique promeut régulièrement l'un des trois personnages-type des opéras au rang 
de "personnage clé ». Dès lors les comparaisons se font de manière non systématique selon les affinités fonctionnelles ou les « profils caractériels » des personnages.

En tout cas, la lecture à partir des rôles vocaux est largement révolue. Elle a perdu son utilité: un très petit nombre d'opéras sont inlassablement joués et rejoués, la surinterprétation littéraire est devenue un exercice courant ; il n'est plus besoin de guide à la lecture, bien au contraire. Il faut bien admettre que la pré-désignation conventionnelle à partir des rôles vocaux n'existe vraiment, et donc ne fait entièrement partie du pacte narratif ${ }^{42}$ entre auditeur et spectateur, que si ce découpage sémiotique est aussi sémantiquement significatif. Autrement dit, il existe un lien privilégié entre les formules de pré-désignation conventionnelle et le canal dont elles cherchent à faciliter la lecture. Il suffit que l'intérêt « littéraire » des opéras disparaisse pour que les marques laissées dans l'économie narrative perdent toute efficacité. Les formes de travail que fournit chaque spectateur pour produire du sens à partir des traces auditives ou visuelles de l'œuvre qu'il perçoit doivent ainsi être soigneusement distinguées. On le voit, toutes les tentatives pour "armer " une œuvre d'un sens de lecture sont un jour ou l'autre déjouées. En un mot, les contraintes de lecture les plus puissantes ne sont pas à rechercher dans les œuvres, mais chez les spectateurs.

\section{NOTES}

1. Une illustration très remarquable en est donnée par S. Arom, dans Polyphonies et polyrythmies instrumentales d'Afrique Centrale, Paris, SELAF, 1985, 2 vol.

2. Cette appellation, au contraire de l'appellation traditionnelle de "sociologie de la musique » signe la revendication de sa double appartenance à la sociologie et à la musicologie.

3. Histoire de la musique. XVIII. Histoire du public musical, Lausanne, Éditions Rencontre, 1967.

4. C'est ainsi que P.-M. Menger (dans Le paradoxe du musicien, Paris, Flammarion, 1985) définit la production savante contemporaine.

5. Barry S. Brook, La symphonie française dans la seconde moitié du XVIII ${ }^{e}$ siècle, Paris, Institut de musicologie de l'université de Paris, 1962, 3 vol.

6. Il s'agit des différentes parties instrumentales et non de leur forme « résumée ", pour clavier par exemple.

7. D’une façon générale, les époques durant lesquelles le public peu formé élit des genres mixtes ou favorise des compromis avec la culture savante, voient se développer des œuvres faussement élaborées. La longue durée des opéras composés de sections courtes littérairement chainées, est une figure exemplaire de ce compromis.

8. Le terrain de ce prélèvement est fourni par une émission où l'invité (intellectuel, etc.), mis en situation de présentation officielle de ses préférences musicales, a eu le loisir de méditer ses choix, de manière sinon moins artificielle du moins plus approfondie que dans un questionnaire.

9. Le dilettantisme musical de Stendhal, violemment attaqué par $\mathrm{H}$. Berlioz, héraut de la musique pure et de son écoute savante, est une illustration parfaite de ce profil. Voir notamment Vie de Rossini, 1823 ; Vies de Haydn, de Mozart et de Métastase, 1814 ; et Voyage en Italie, Paris, Gallimard, «Bibliothèque de la Pléiade ». Le rôle méthodologiquement privilégié des formes « impures » ou «mixtes» (polysémiotiques) dans l'exploration sociologique des «pactes de réception» est 
souligné, à propos de la littérature et de la peinture, par J.-C. Passeron («La perception esthétique : questions sociologiques », EHESs, Séminaire 1986-87).

10. La possibilité de manipuler dans le détail le flux musical ininterrompu - lorsque l'œuvre est longue - fait naturellement défaut à ceux qui sont dépourvus d'instruments analytiques proprement musicaux, d'où une prédilection pour toutes les formes aisément "pilotables » par un texte ou suffisamment courtes pour éviter un travail de repérage.

11. Il y a, dans les analyses littéraires des livrets d'opéra, quelques beaux exemples de «surinterprétation» (cf. J.-C. Passeron («La perception esthétique...», EHEss, Séminaire 1986-87).

12. Il n'existe pas actuellement de bonne histoire des publics, je le regrette.

13. H. Berlioz, Mémoires (1870), Paris, Garnier-Flammarion, rééd. 1959, t. 1.

14. Soulignons l'importance quantitative des commentaires sur les musiques populaires. Pour la vie musicale urbaine, les descriptions portent essentiellement sur les opéras, à l'exception d'un concert de musique religieuse à Rome.

15. Berlioz, Mémoires, p. 214, 215 et216.

16. Au début du XIX ${ }^{e}$ siècle, les orchestres italiens ainsi que l'enseignement musical sont en totale décomposition. Le bilan que donne H. Berlioz n'est pas excessif, cf. G. Monaldi, «Orchestre e direttori del secoloxIX », Rivista musicale italiana, XVI, 1909, p. 123.

17. Berlioz, Mémoires, p. 246, 247 et 248.

18. Ibid., p. 260.

19. Ibid., p. 276-277.

20. Dans de très nombreuses villes, il existe, de fait, un monopole de la maison Ricordi. À partir de 1850, un monopole de droit, pour l'ensemble du territoire italien, remplace la situation de fait. Le travail de recherche est évidemment plus simple qu'en France.

21. La musique instrumentale s'est très peu implantée en Italie au début du siècle, elle ne réapparaîtra qu'à l'extrême fin du $\mathrm{XIX}^{\mathrm{e}}$ siècle. Pourtant «l'éclipse que subira la musique instrumentale ", selon l'expression de R. Di Benedetto, au profit de la musique vocale suit une période particulièrement riche et influente. «On sait, en effet, combien les œuvres de Haydn et de Mozart sont nourries de sève italienne : non seulement la musique vocale, où l'influence de l'école napolitaine fut prépondérante - elle connaît une troisième et dernière floraison éblouissante avec N. Piccini, G. Paisiello, A. Sacchini, D. Chimarosa - mais également la musique instrumentale. Rappelons la contribution de G.B. Sammartini à la formation de la symphonie moderne, puis le développement de la littérature galante (sonate), dont les maîtres furent G.B. Platti, G.M. Rutini, P.D. Paradisi. » (R. Di Benedetto, « La musique italienne », in M. Honegger, ed., Dictionnaire de la musique. Science de la musique, I. Les techniques, Paris, Bordas, 1976, p. 520.)

22. Deux «pointages» peuvent être effectués. En 1739, le président de Brosses donne une description des publics de l'opéra qui correspond du reste à ce que nous en savons par ailleurs : le public aristocratique, pour lequel ces manifestations sont montées, est peu mêlé, alors que le parterre abondant et très présent se compose du " petit peuple », la « racaille » selon l'expression du président de Brosses. En 1814, Stendhal observe que, alors que les parterres sont vides, la société aristocratique et bourgeoise se partage les loges de la Scala de Milan devenue le grand salon de la ville.

23. W. Weber, "Mass culture and the reshaping of European musical taste 1770-1870", International Review of the Aesthetics and Sociology of Music, VIII (1), juin 1977, p. 5-20.

24. F. Dutheil, Le livret d'opéra italien, 1825-1850, Bordeaux, Université Bordeaux-III, 1985, thèse d'État, 4 vol.

25. La formation musicale de G. Verdi est exemplaire: issu d'un milieu populaire rural, sa formation musicale reste provinciale et largement incomplète. Les biographies en profitent naturellement pour créditer G. Verdi d'un génie inversement proportionnel à sa formation visible. 
26. P. Cambiasi, Rappresentazioni date nei reali teatri di Milano, 1772-1872. Opere in musica, nome dei maestri, poeti ed esecutori principali, data della prima rappresentazione e classificazione dell'esito, balli, ecc., Bologne, Forni, 1872.

27. Autrement dit, on ne compare plus des éléments comparables, si on lit la troisième ligne du tableau, croyant déceler une relative stabilité dans la production lyrique.

28. La littérature musicologique spécialisée s'est empressée de qualifier cette abondante production d'opéras de "première culture de masse", atténuant les distances sociales: "le caractère de culture de masse de l'opéra est confirmé par les chiffres : à côté du quatuor des "grands" - Rossini, Bellini, Donizetti, Verdi - on trouve une foule d'auteurs secondaires comme G. Mercadante, G. Pacini, N. Vaccal, L. et F. Ricci, qui doivent travailler sans relâche pour satisfaire les demandes incessantes du marché » (R. DiBenedetto, «La musique italienne », P. 540). Or nous retrouvons les mêmes caractéristiques un siècle auparavant, pour un opéra toujours très productif qui savait rassembler un éventail social plus étendu comme nous l'avons souligné (cf. supra n.22).

29. F. Dutheil, Le livret d'opéra italien. À Milan en 1840 l'on vend plus de livrets, digest de la littérature européenne, que de traductions, alors diffusées confidentiellement.

30. Il n'est pas rare de retrouver dans les archives les collections de livrets reliées aux armes d'une famille importante. Notons que la pratique de la reliure d'opuscules de nos jours condamnés à la dégradation immédiate était courante. Les journaux quotidiens étaient ainsi régulièrement reliés.

31. Les discussions portent sur les situations; l'auteur eut-il raison de faire mourir tel ou tel personnage à tel moment ou, ce que dit tel ou tel personnage quant à sa situation est-il judicieux, etc.

32. Les œuvres qui nous occupent sont en outre musicalement très accessibles. Classé, par la grande majorité des musicologues, au plus bas - à quelques exceptions près - de la production musicale harmonique homophone du XIx ${ }^{\mathrm{e}}$ siècle, l'opéra est également et couramment décrit par des caractéristiques négatives, stéréotypie harmonique, absence de plan tonal, pauvreté des modulations, extrême rareté - pour ne pas dire absence - de polyphonie contrapuntique, etc. que l'on retrouve à l'inverse, et toujours positivement, pour la musique instrumentale. Il reste que l'« opéra romantique italien » a importé, avec retard, les inventions de la musique harmonique européenne; l'opéra s'est ainsi constamment réactualisé sans pour autant transformer son contenu. Enfin, il ne fait pas de doute que l'«opéra romantique italien", contrairement à la symphonie ou au quatuor, est une fausse "grande forme "; composée de courtes sections (airs, trios, etc.) littérairement accolées les unes aux autres, l'œuvre lyrique ne possède pas de plan tonal à proprement parler. C'est par excès de langage que l'on dit que les sections s'enchaînent musicalement, il est plus exact de remarquer qu'elles ne sont pas incompatibles tonalement, qu'il n'existe pas de brusques « sauts » de tonalité. Il en découle que le « guide » de la longue durée de l'œuvre n'est pas strictement musical.

33. Les livrets sont naturellement très peu homogènes. Leur longueur moyenne est d'une quarantaine de pages. La partie strictement narrative (fonctionnelle) est limitée, très approximativement, à dix à quinze pour cent du livret. Les « péroraisons » multiples meublent par ailleurs une bonne partie du texte restant. Ces deux types de texte ne sont pas soumis à un même mode d'écriture : la versification est certes continue mais les "archaïsmes " littéraires sont plus fréquents dans les séquences non fonctionnelles.

34. Voir, sur une question similaire, U. Eco, « James Bond, une combinatoire narrative », Poétique, 19, 1974.

35. P. Hamon, « Pour un statut sémiologique du personnage », Lectures, 1972, p. 86-110.

36. Ibid., p. 93.

37. Les trois personnages principaux totalisent plus de $90 \%$ du temps d'intervention vocale des personnages individualisés. 
38. La chanson, proche à certains égards de l'opéra, a été et est sporadiquement susceptible d'utiliser ces procédés conventionnels. L'opposition timbre masculin vs timbre féminin est courante: l'opposition de registre advient parfois mais dépasse rarement l'opposilion binaire (grave-aigu). Il en va de même pour la comédie musicale. La double opposition sexuelle et tessiturale est a fortiori encore plus rare.

39. La distinction de la voix de mezzo soprano date de 1838.

40. P. Hamon, « Pour un statut sémiologique... », p. 96.

41. La parenté entre opéra et conte tient au mode de transmission de ces formes. R. Courtès a bien analysé les caractéristiques sémiotiques liées à la transmission des contes : «C'est [...] le propre des contes (et ce qui, comme dans le mythe, en assure la transmissibilité) que d'accuser des rapports d'oppositions très nets (ex : les bons d'un côté, les méchants de l'autre). Certes l'on peut très bien concevoir entre eux des caractères intermédiaires, tels qu'on en trouve par exemple dans une nouvelle ou un roman : mais il est clair que si, dans ce cas, les oppositions y sont affaiblies ou plus réduites que sur un axe sémantique donné, elles se trouvent comme multipliées en contrepartie par une diversification des plans isotopiques (correspondant aux différentes lectures possibles). Quant au conte populaire qui, pour sa part, ne peut jouer sur de nombreuse isolopies et leurs corrélations (ce qui demanderait au conteur une mémoire prodigieuse), il ne saurait préserver sa spécificité et sa survie s'il se laissait aller aux nuances. » (R. Courtès, "De la description à la spécificité du conte populaire merveilleux français", Ethnologie française, nouv. série, 2 (1), 1972, p. 15.)

42. Il a été largement débattu de l'utilisation sociologique de ce concept dans le séminaire de J.C. Passeron (1986-87). 